\author{
C. Knittlmayer · H.-J. Muffler • Ch.-H. Fischer • \\ W. Weppner
}

\title{
Investigation of electrochromic tungsten trioxide thin films prepared by the ILGAR method
}

Received: 19 April 2006 / Accepted: 28 April 2006 / Published online: 10 June 2006

C) Springer-Verlag 2006

\begin{abstract}
An ion layer gas reaction dip coating process for the deposition of tungsten trioxide has been developed. Thin films of electrochromic tungsten trioxide with thicknesses of up to $150 \mathrm{~nm}$ were prepared. The films were found to be microcrystalline by X-ray diffraction analysis. The growth rate of the films was measured by profilometry. The chemical diffusion coefficient of lithium was investigated as a function of the concentration of lithium by the electrochemical galvanostatic intermittent titration technique. The chemical diffusion coefficient $\widetilde{D}$ was found to increase slightly from $7 \times 10^{-12}$ to $3 \times 10^{-1} \mathrm{~cm}^{2} / \mathrm{s}$, with $x$ increasing from 0.2 to 0.8 in $\mathrm{Li}_{x} \mathrm{WO}_{3}$.
\end{abstract}

Keywords Electrochromics - Thin film ionics · Tungsten trioxide $\cdot$ ILGAR

\section{Introduction}

The electrochromism of thin films of tungsten trioxide was first studied by T. Kraus in 1953 [1]. The term "electrochromism" was introduced by J. Platt in 1961 [2]. Since that time, many different materials have been considered for electrochromic devices. Nevertheless, in spite of all these efforts, $\mathrm{WO}_{3}$ remained the best candidate as electrochromic cathode during coloration in three-layer galvanic cell arrangements.

C. Knittlmayer · W. Weppner $(\bowtie)$

Sensors and Solid State Ionics, Faculty of Engineering,

Christian-Albrechts-University,

Kaiserstr.2

24143 Kiel, Germany

e-mail: ww@tf.uni-kiel.de

H.-J. Muffler · C.-H. Fischer

Hahn-Meitner-Institute,

SE 2, Glienickerstr. 100,

14109 Berlin, Germany
One of the most critical aspects of the fabrication of electrochromic devices is the formation of the thin films. Several papers have described the present state of fabrication of such tungsten trioxide films for electrochromic applications [3]. Thin films of $\mathrm{WO}_{3}$ were typically deposited by evaporation [4], sputtering [5], electrodeposition [6], and sol-gel method [3, 7, 8].

Especially, the physical vacuum thin film deposition techniques, e.g., such as sputtering and evaporation are very costly. In contrast, chemical thin film deposition techniques, e.g., chemical bath deposition, spray pyrolysis, sol-gel, or electrodeposition, have comparatively low costs. Despite of their merits, all these techniques have several disadvantages such as waste of reagent or difficulties to control the film thickness.

In the present work, the preparation of tungsten trioxide by ion layer gas reaction (ILGAR) [9] was investigated. This technique was originally introduced for the preparation of sulfides such as $\mathrm{CuInS}_{2}$ for absorber layers in extremely thin absorber solar cells and for $\mathrm{CdS}$ or $\mathrm{ZnS}$ being used as buffer layers in chalcopyrite heterojunction solar cells [10]. Later, this method was extended to the deposition of metal oxides such as $\mathrm{ZnO}[11,12]$.

The ILGAR method offers several further advantages such as coating of dense and porous substrates of nearly any shape, uncritical process parameters, deposition of doped materials, and formation of multilayers. Fully automated, ILGAR is an extreme low-cost process since no complicated setup such as vacuum equipment has to be used. The method can be generally applied for the deposition of other hardly soluble oxides.

\section{Experimental description}

The sequential cyclic process of the deposition of thin film tungsten trioxide consists of the following steps:

1. First step: A precursor solution containing tungsten hexachloride in an organic solvent is deposited by dipping. 
Fig. 1 GID-XRD analysis of tungsten trioxide thin films prepared by ILGAR compared to JCPDS 73-2182. 1 Sample prepared at $200{ }^{\circ} \mathrm{C}$ reaction temperature (30 cycles), 2 sample prepared at $400{ }^{\circ} \mathrm{C}$ reaction temperature (30 cycles), 3 sample prepared at $400{ }^{\circ} \mathrm{C}$ reaction temperature ( 30 cycles) and subsequently annealed in air at $400{ }^{\circ} \mathrm{C}$ for $1 \mathrm{~h}$

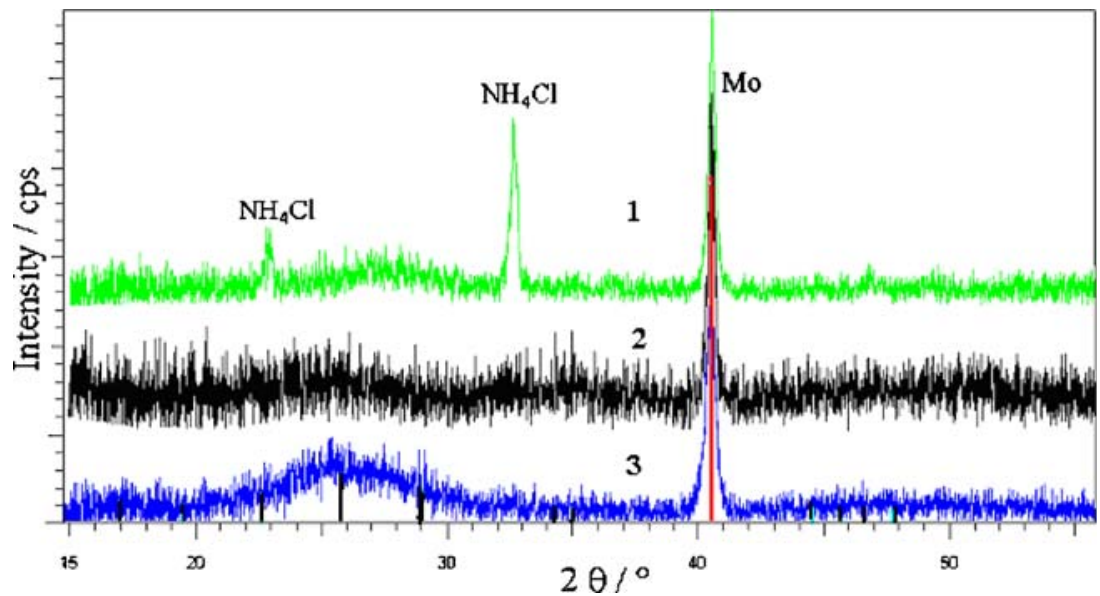

2. Second step: After drying, the precursor film is converted into tungsten hydroxide with wet ammonia gas at temperatures between 100 and $400{ }^{\circ} \mathrm{C}$ according to

$$
\mathrm{WCl}_{6}+6 \mathrm{NH}_{3}+6 \mathrm{H}_{2} \mathrm{O} \rightarrow W(\mathrm{OH})_{6}+6 \mathrm{NH}_{4} \mathrm{Cl}
$$

Finally, tungsten trioxide is formed by decomposition in air:

$$
W(\mathrm{OH})_{6}+\stackrel{\Delta}{\longrightarrow} \mathrm{WO}_{3}+3 \mathrm{H}_{2} \mathrm{O}
$$

3. Third step: The film is rinsed in organic solvents to remove undesirable by-products.

These steps are repeated until the desired film thickness is reached. The whole process is automated by using an $x$-, $y$-, and $z$-robot. The initial growth rate per cycle depends on the substrate. If the precursor solution remains the same, it is constant as soon as a closed cover layer is formed. Therefore, the film thickness can be easily controlled and reproduced.

Three different types of substrates were used: float glass with a size of $1 \times 1$ in. to investigate mainly the growth rate, molybdenum thin films for X-ray diffraction (XRD) measurements and platinum thin films with a size of
$3.5 \times 3.5 \mathrm{~cm}$ for electrochemical investigations. All substrates were cleaned with isopropanol in an ultrasonic bath before deposition.

Forty millimolar of tungsten hexachloride $\left(\mathrm{WCl}_{6}, 99.9 \%\right.$ Aldrich) in tetrahydrofurane (THF, 99.8\% Merck) was used as precursor solution. The ammonia/water reaction gas was generated by bubbling nitrogen through a $25 \%$ aqueous ammonia solution. The samples were reacted during every cycle at temperatures between 100 and $400{ }^{\circ} \mathrm{C}$ for $30 \mathrm{~s}$ and, afterwards, were cleaned in THF to remove the by-product ammonium chloride or uncompletely converted starting material.

XRD was determined by means of a "Bruker Advance D8" diffractometer.

Electrochemical measurements were carried out using a computer controlled galvanostat/potentiostat (IonicSystems, Stuttgart, PG 1.0) in galvanostatic mode.

\section{Results}

X-ray diffraction analysis

To analyze the optimum process conditions for the deposition of ILGAR $\mathrm{WO}_{3}$ layers, three samples were
Fig. 2 Growth rate of ILGAR $\mathrm{WO}_{3}$ as determined by a profilometer using float glass as substrate

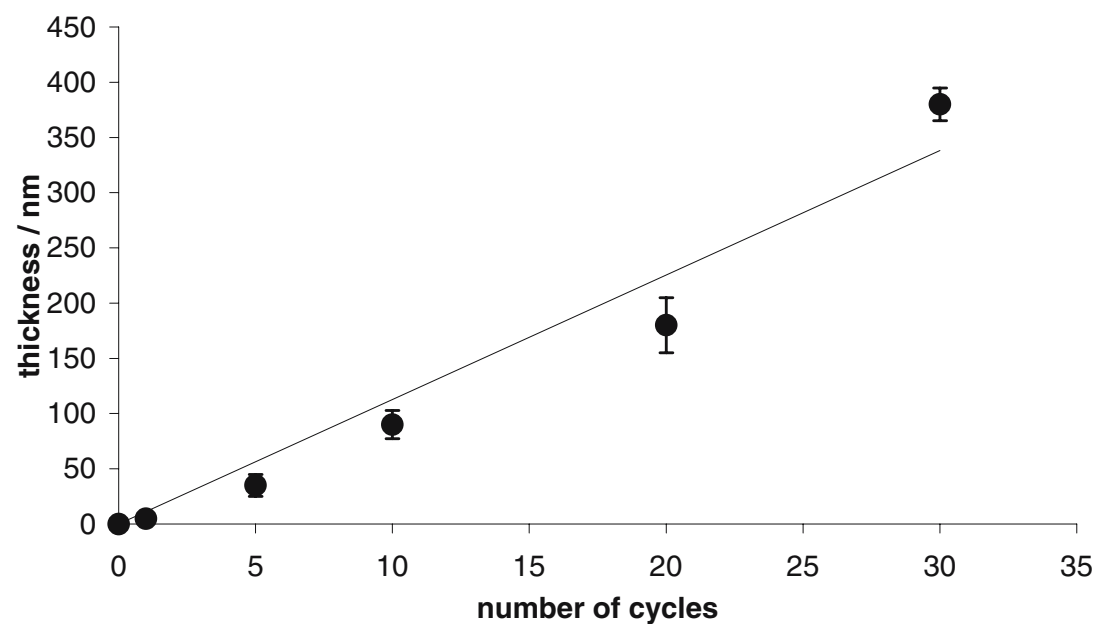


Fig. 3 Galvanic cell arrangement for electrochemical investigations, operated inside an argon-filled glove box

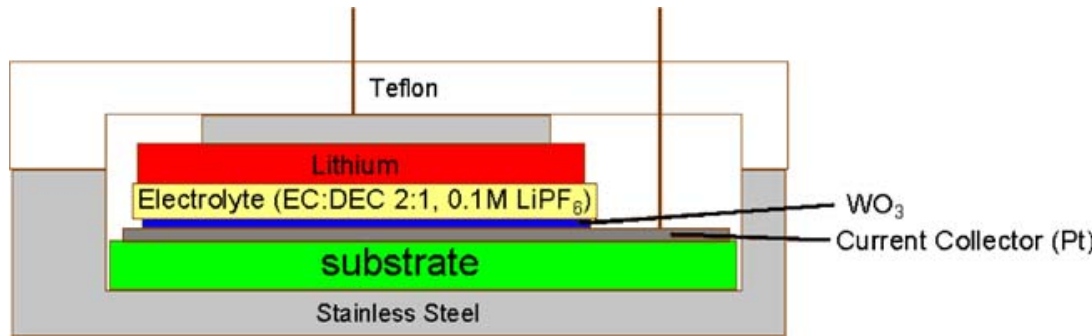

prepared within 30 cycles using molybdenum thin film substrates. Sample 1 was processed at a reaction temperature of $200^{\circ} \mathrm{C}$ for $30 \mathrm{~s}$ each cycle, sample 2 at $400{ }^{\circ} \mathrm{C}$, and sample 3 also at $400{ }^{\circ} \mathrm{C}$ but with subsequent annealing at $400{ }^{\circ} \mathrm{C}$ for $1 \mathrm{~h}$. The XRD patterns (Fig. 1) of these samples were taken in grazing incidence mode $(\omega=0.3)$.

Sample 1 shows an X-ray amorphous behavior of the $\mathrm{W}$-containing component. The two observable signals could be assigned to a cubic phase of ammonium chloride according to JCPDS 73-0365. These signals disappear for sample 2, when ammonium chloride had sublimed at the higher temperature of $400^{\circ} \mathrm{C}$. The XRD pattern of sample 3 shows a broad signal, which can be assigned to the monoclinic phase of $\mathrm{WO}_{2.9}$ according to JCPDS 73-2182.

\section{Growth rate by profilometry}

To determine the thickness and growth rate, profilometric measurements of samples deposited on to a float glass were carried out using a profilometer (Veeko DEKTAK 3 ST). All samples prepared for these profilometric measurements were reacted at $400{ }^{\circ} \mathrm{C}$ for $30 \mathrm{~s}$ each cycle. In Fig. 2, the film thickness is plotted as a function of the number of deposition cycles. From the slope of the linear fit, a growth rate of $12 \mathrm{~nm} /$ cycle is determined. The variation in the thickness measured by the profilometer $\left(\mathrm{RP}_{05}\right)$ is indicated by the error bars.

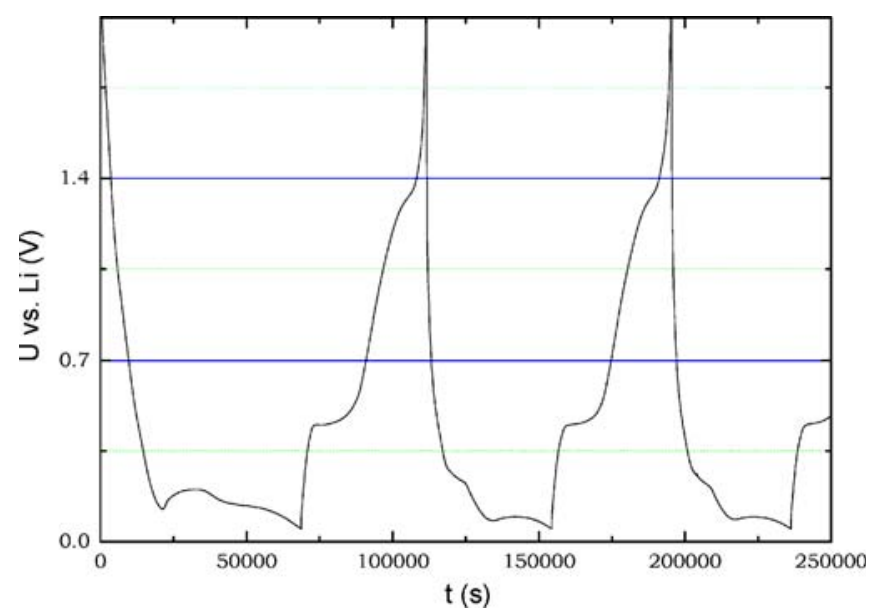

Fig. 4 Charge and discharge curves under a current of $I=0.1 \mathrm{~mA}$
Electrochemical investigation

All electrochemical investigations were carried out employing samples, which were deposited on to Pt thin films with a film thickness of about $150 \mathrm{~nm}$. For this investigation, a computer controlled galvanostat/potentiostat (Ionic-Systems, PG 1.0) was used in the galvanostatic mode. The electrochemical measurements were performed in an argon-filled glove box. To avoid influences of the atmosphere, a cell setup, as shown in Fig. 3, was used.

The sample was housed in a stainless steel container. A microporous membrane soaked in with commercial "Merck LP30" electrolyte was placed onto the sample. A small sheet of lithium foil was applied as counter electrode. After assembling, the cell was closed with a Teflon cap. The cell arrangement was loaded for better contact with a stainless steel cover. The sample was connected to the outer circuit by a spring-loaded gold pin fed through a small hole in the cap.

The cell was cycled six times between 2.0 and $0.1 \mathrm{~V}$. It was opened after the fifth lithiation of $\mathrm{WO}_{3}$. As expected, the former colorless film showed a deep blue color. After assembling the cell again and delithiation, the coloration had disappeared.

Figure 4 shows the charging and discharging curves of the first and second cycle. During the intercalation of lithium two 2-phase regions appear: the first one at $1.2 \mathrm{~V}$

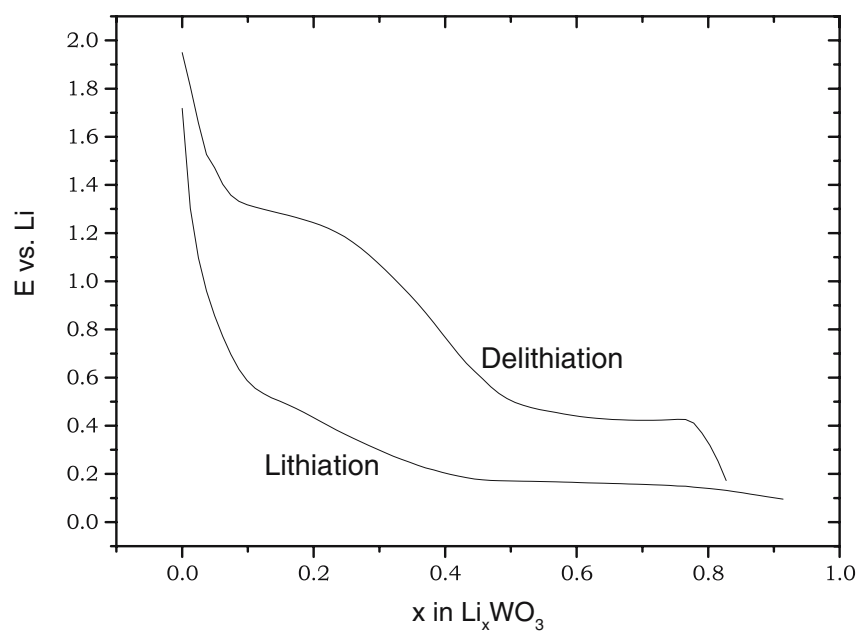

Fig. 5 Open-circuit voltages of the coulometric titration of $\mathrm{Li}^{+}$into and out of $\mathrm{Li}_{x} \mathrm{WO}_{3}$ 


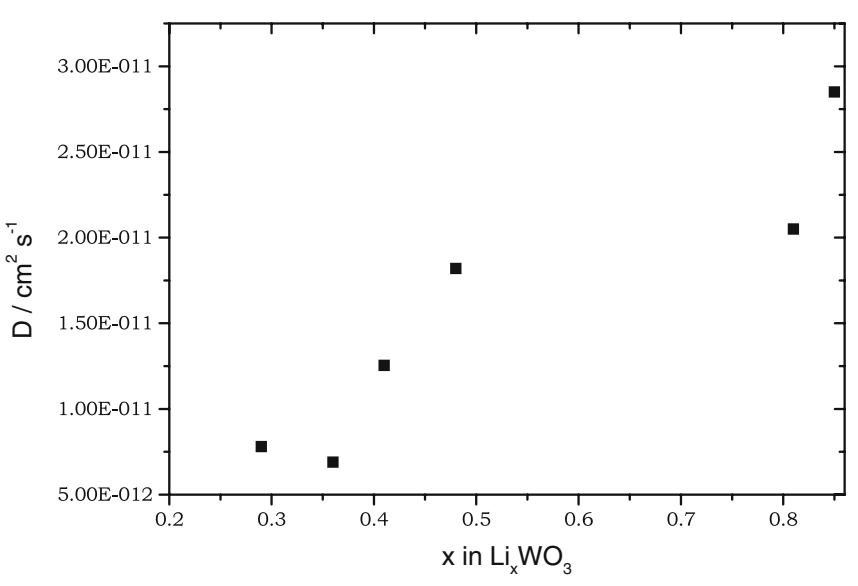

Fig. 6 Chemical diffusion coefficient $\widetilde{D}$ of $\mathrm{Li}$ in $\mathrm{Li}_{x} \mathrm{WO}_{3}$ as determined by GITT

and the second one at $0.6 \mathrm{~V}$. The curves agree well with the data of Avellaneda and Bulhões [13].

To obtain the chemical diffusion coefficient $\widetilde{D}$ of $\mathrm{Li}$ in $\mathrm{WO}_{3}$ and $\mathrm{Li}_{x} \mathrm{WO}_{3}$, the galvanostatic intermittent titration technique (GITT) [14] was applied. Figure 5 shows the coulometric titration curves for lithiation up to $x=0.8$ in $\mathrm{Li}_{x} \mathrm{WO}_{3}$ and subsequent delithiation. The chemical diffusion coefficients are shown in Fig. 6.

\section{Discussion}

Both the lithiation and the delithiation curve under current (Fig. 4) and the open circuit measurements (Fig. 5) exhibit voltage shoulders. Curves of this shape are typically observed in microcrystalline materials ([1], pp 86-88).

Because $\mathrm{Li}_{x} \mathrm{WO}_{3}$ may be considered to be a solid solution of $\mathrm{Li}$ in $\mathrm{WO}_{3}$, we may assume that the number of electrons determines largely the shape of the curves. The lithiation curve in Fig. 5 indicates a small number of electrons initially. The slope becomes smaller and smaller due to the increasing number of electrons as a result of the increase of $x$ and the increasing concentration of electrons in $\mathrm{Li}_{x} \mathrm{WO}_{3}$.

The delithiation was assumed to be completed when the voltage reached $2 \mathrm{~V}$ under current. The deviation between the lithiation and delithiation curves is assumed to be due to the small thickness of the $\mathrm{WO}_{3}$ layer and the possibility that lithium ions are absorbed by the Pt used as current collector as well as by the glass substrate and kinetic impedances. Furthermore, the amount of organic electrolyte is large compared to the amount of $\mathrm{WO}_{3}$ in the sample. With a density of $7.16 \mathrm{~g} / \mathrm{cm}^{3}$ for monoclinic tungsten trioxide ([1], p 31), the mass ratio of active material to electrolyte was calculated to be in the range of $\mathrm{M}_{\mathrm{WO}}$ : $\mathrm{M}_{\mathrm{el}}=3 \times 10^{-6}$. This means that a small variation of the stoichiometry of the electrolyte in the range of $10^{-4} \%$ corresponds to a change of $x$ in $\mathrm{Li}_{x} \mathrm{WO}_{3}$ of $x=0.06$.

The values of the chemical diffusion coefficient $\widetilde{D}$ of $\mathrm{Li}$ (Fig. 6) increases slightly with the increase of lithium in $\mathrm{Li}_{x} \mathrm{WO}_{3}$. That may be explained by the fact that $\mathrm{Li}^{+}$is increasingly driven by the simultaneously increasing concentration of electrons for balancing the charge of the mobile ions.

Assuming a film thickness of $l=150 \mathrm{~nm}$ and a chemical diffusion coefficient of $\widetilde{D}=10^{-1} \mathrm{~cm}^{2} / \mathrm{s}$, the time $\tau$ to penetrate this layer completely is in the order of $10 \mathrm{~s}$ according to $\tau=l^{2} / 2 \widetilde{D}$.

\section{Conclusion and summary}

A new method for the preparation of electrochromic $\mathrm{WO}_{3}$ thin films was presented. The ion layer gas reaction (ILGAR) technique is fast, reliable, simple, and cheap. The technique can also be carried out by spraying instead of dipping and is then perfectly suited for industrial in-line processes.

The obtained $\mathrm{WO}_{3}$ films show a reversible electrochromic effect upon the exchange of lithium. The chemical diffusion coefficient $\widetilde{D}$ of lithium in these films was determined to be in the range of $10^{-1} \mathrm{~cm}^{2} /$ s. The value of $\widetilde{D}$ increases slightly with the degree of lithiation. The originally colorless films turned blue at a stoichiometry of $x=0.3-0.4$ in $\mathrm{Li}_{x} \mathrm{WO}_{3}$.

\section{References}

1. Granqvist CG (1995) Handbook of inorganic electrochromic materials. Elsevier, Amsterdam

2. Platt JR (1961) J Chem Phys 34:862

3. Avellaneda CO, Bulhões LOS (2003) J Solid State Electrochem 7:183-186

4. Bohnke O, Bohnke C, Robert G, Carquille B (1982) Solid State Ion $6: 121$

5. Masetti E, Grilli ML, Dautzenberg G, Macrelli G, Adamik M (1999) Sol Energy Mater Sol Cells 56:259-269

6. Meulenkamp EA (1997) J Electrochem Soc 144:1664-1671

7. Chemseddine A, Morineau R, Livage J (1983) Solid State Ion 9-10:357

8. Xu G, Chen L (1988) Solid State Ion 28-30:1726

9. Fischer CH, Möller J, Krönkamp R, Lux-Steiner M, Siebentritt S (1999) German Patent DE 198 31:214, Hahn-Meitner-Institut, Berlin

10. Möller J, Fischer CH, Könenkamp R, Kaiser I, Kelch C, LuxSteiner MC (2000) Thin Solid Films 361-362:113-117

11. Fischer CH, Muffler HJ, Lux-Steiner MC (1999) German Patent DE 199 16:403, Hahn-Meitner-Institut, Berlin

12. Bär M, Muffler HJ, Fischer CH, Lux-Steiner MC (2001) Sol Energy Mater Sol Cells 67:113-120

13. Avellaneda CO, Bulhões LOS (2003) Solid State Ion 165: 59-64

14. Weppner W, Huggins RA (1977) J Electrochem Soc 124:1596 